CITUSC/00-065

hep-th/0012180

\title{
ON RELATIVISTIC BRANE PROBES IN SINGULAR SPACETIMES
}

\author{
P. Berglund \\ CIT-USC Center for Theoretical Physics \\ Department of Physics and Astronomy \\ University of Southern California \\ Los Angeles, CA 90089-0484 \\ T. Hübsch 目 \\ Department of Physics and Astronomy \\ Howard University \\ Washington, DC 20059 \\ D. Minic日 \\ CIT-USC Center for Theoretical Physics \\ Department of Physics and Astronomy \\ University of Southern California \\ Los Angeles, CA 90089-0484
}

The brain is drained

And a brown ament, and the noun I meant

To use but did not, dry on the cement.

- John Francis Shade

\begin{abstract}
We study the relativistic dynamics of brane probes in singular warped spacetimes and establish limits for such an analysis. The behavior of the semiclassical brane probe wave functions implies that unitarity boundary conditions can be imposed at the singularity.
\end{abstract}

\footnotetext{
${ }^{1}$ e-mail: berglund@citusc.usc.edu

${ }^{2}$ e-mail: thubsch@howard.edu

${ }^{3}$ On leave from the "Rudjer Bošković" Institute, Zagreb, Croatia.

${ }^{4}$ e-mail: minic@citusc.usc.edu
} 


\section{Introduction}

Various brane configurations in string theory describe spacetime geometries which possess repulsive singularities [1]. These repulsons can be resolved in string theory via the enhançon

mechanism [2, 3, 4, 5, 6, 7], which describes the constituent branes that effectively repel each other, thus forming a nonsingular matter shell of finite radius. The original enhançon of Ref. [2] involved wrapping $N D p$ branes on a $K 3$ manifold, the physics of which is described by large $N$ gauge theories with eight supercharges. The naive singular repulson geometry is replaced by a non-singular configuration of finite radius $r_{e}$, where the tension of the wrapped branes vanishes. This mechanism is related to the appearance of a negative number of induced $(p-4)$-branes upon wrapping the $p$-branes on the $K 3$ manifold [8].

Recently, we have discussed an analogue of the enhançon mechanism in a non-supersymmetric set-up [9], which involved space-time varying string vacua [10, 11] with exponentially large hierarchy [12, 13]. Continuing this work, in this note we use the Dirac-BornInfeld action [14, 15] to describe a relativistic probe analysis of a class of singular spacetime geometries including both repulsive and attractive naked singularities. We discuss the familiar supersymmetric (BPS) examples, and certain non-supersymmetric generalizations [12, 16, 17, 18]. Furthermore, we find that the behavior of the semiclassical brane probe wave functions suggests that unitarity boundary conditions can be imposed at the singularity. We briefly discuss the relation of this result to various prescriptions for the resolution of singularities [19, 20]. Our analysis is also consistent with the generic resolving properties of $D$-brane probes [21, 22].

Throughout, we consider spacetime geometries where the metric is constant in the $p$ 'longitudinal' (brane) directions and varies over the 'transverse' space, but in a 'spherically' symmetric way. That is, we assume the spacetime metric - in the Einstein frame-to be of the type

$$
\mathrm{d} s_{E}^{2}=e^{2 A(r)} \eta_{a b} \mathrm{~d} x^{a} \mathrm{~d} x^{b}+e^{2 B(r)}\left(\mathrm{d} r^{2}+r^{2} \mathrm{~d} \vec{\Omega}_{8-p}^{2}\right)
$$

where $a, b=0, \cdots, p$ and $\mathrm{d} \vec{\Omega}$ is the transverse 'spherical' angle element. The explicit forms of the warp factors in various applications are listed in section 3 .

\section{Relativistic brane probe dynamics}

In this section, we will derive a band of allowed energies for relativistic $D p$-brane probes in warped singular spacetimes, and a WKB estimate for their wave-functions. These wavefunctions will be used to argue the existence of unitarity boundary conditions at the singularity. 
The action functional that is used to determine the dynamics of a $D p$-brane probe in a given background is the Dirac-Born-Infeld action [14, 15]:

$$
S_{p}=-\tau_{p} \int \mathrm{d}^{p+1} \xi e^{-\Phi} \sqrt{\operatorname{det}\left[G_{a b}^{s}+B_{a b}+2 \pi \alpha^{\prime} F_{a b}\right]}+\mu_{p} \int \mathrm{d}^{p+1} \xi C_{p+1},
$$

where $\tau_{p}=\mu_{p} g_{s}^{-1}$ and $\mu_{p}=2 \pi\left(2 \pi \sqrt{\alpha^{\prime}}\right)^{-(p+1)}$ are the brane probe's tension and charge, respectively.

Aligning the brane coordinates with the spacetime ones, $\xi^{0}=t$ and $\xi^{a}=x^{a}, a=1, \cdots, p$, induces the metric on the brane probe from that on the spacetime. For diagonal spacetime metrics (1.1), the metric induced on a $D p$-brane is, in the string frame,

$$
\left[G_{a b}^{s}\right]=\operatorname{diag}[-\left(e^{2 A+\Phi / 2}-e^{2 B+\Phi / 2} v^{2}\right), \underbrace{e^{2 A+\Phi / 2}, \cdots, e^{2 A+\Phi / 2}}_{p}]
$$

Furthermore, herein we focus on cases with $B_{a b}=0=F_{a b}$, which simplifies the action (2.1) to that of a relativistic 'particle':

$$
S_{p}=\int \mathrm{d}^{p+1} \xi \mathcal{L}, \quad \mathcal{L}=-m c^{2} \sqrt{1-\frac{v^{2}}{c^{2}}}-\mathcal{V},
$$

where

$$
c=e^{A-B}, \quad m=\tau_{p} e^{\frac{p-3}{4} \Phi} e^{(p-1) A+2 B}, \quad \mathcal{V}=-\mu_{p} C_{p+1}
$$

all depend on $r$, and can be interpreted as the 'effective' speed of light, mass and potential for the brane probe, respectively. We first derive several results in this 'general' notation, and then apply these to brane probes of various codimensions.

\subsection{Limits on the brane probe's total energy}

Given the Lagrangian (2.3), the total energy is obtained through the Legendre transform

$$
\mathcal{E}=\frac{m c^{2}}{\sqrt{1-v^{2} / c^{2}}}+\mathcal{V}
$$

Using the conservation of total energy, Eq. (2.5) can be solved to express:

$$
v^{2}=c^{2}\left[1-\frac{m^{2} c^{4}}{(\mathcal{E}-\mathcal{V})^{2}}\right] \text {. }
$$

\footnotetext{
${ }^{5}$ The Legendre transform is of course performed with respect to all 'spherical' coordinates; the final result is re-expressed in terms of the 'total' speed, $v$. Throughout this note, we will explicitly write formulae valid for the case of codimension-2; higher codimension generalizations are easy to generate.
} 
Requiring this to be positive, we obtain the lower bound on the total energy (density):

$$
\mathcal{E} \geq m c^{2}+\mathcal{V}=\tau_{p} e^{\frac{p-3}{4} \Phi} e^{(p+1) A}-\mu_{p} C_{p+1} .
$$

The 'non-relativistic' (small $v / c$ ) expansion of Eq. (2.3) is

$$
\mathcal{L} \approx \frac{1}{2} m v_{r}^{2}-\left(m c^{2}+\mathcal{V}-\frac{\ell^{2}}{2 m r^{2}}\right) \text {. }
$$

Of course, when $v / c \sim 1$, the Lagrangian (2.8) is no longer reliable. In addition, the family of Lagrangians (2.3) omit various other effects (perturbation of the background by the probe, back-reaction, radiation, etc.). It seems plausible that these effects are less and less negligible as $v / c \rightarrow 1$, but there is no strict critical value of $v / c$ where the family of Lagrangians (2.8) or even their 'exact' counterparts (2.3) should abruptly become incomplete.

However, one expects that the discrepancy between the 'relativistic' and the 'nonrelativistic' kinetic energy should be bounded by a multiple of the latter:

$$
\mathcal{T}-\mathcal{T}_{N R} \leq \nu \mathcal{T}_{N R}, \quad \text { i.e., } \quad \mathcal{T} \leq(1+\nu) \frac{1}{2} m v^{2}, \quad \nu>0 .
$$

Using Eq. (2.5), this requirement yields:

$$
(1+\nu) \frac{1}{2} m v^{2} \geq \mathcal{E}-\left.\mathcal{E}\right|_{v=0}=m c^{2}\left[\frac{1}{\sqrt{1-v^{2} / c^{2}}}-1\right]
$$

which is a cubic inequality for $v / c$. This reproduces the $v^{2} \geq 0$ inequality which led to (2.7), and produces two more limiting values one of which is negative and so unphysical. The third inequality reads:

$$
\frac{v}{c} \leq \sqrt{\frac{\sqrt{(\nu+1)(\nu+9)}+\nu-3}{2(\nu+1)}}\left\{\begin{array}{ll}
=0 & \text { if } \nu=0, \\
\approx 0.786 & \text { if } \nu=1, \\
=1 & \text { if } \nu=\infty
\end{array} .\right.
$$

Clearly, the 'ultra-relativistic' case implies an infinite discrepancy between the 'relativistic' and 'non-relativistic' kinetic energies. We will hereafter use the $\nu=1$ limit. When inserted in Eq. (2.5), this produces an upper limit on the total energy (density):

$$
\begin{aligned}
& \mathcal{E} \leq \beta m c^{2}+\mathcal{V}=\beta \tau_{p} e^{\frac{p-3}{4} \Phi} e^{(p+1) A}-\mu_{p} C_{p+1}, \\
& \beta=\sqrt{\frac{2(\nu+1)}{\nu+5-\sqrt{(\nu+1)(\nu+9)}}} \approx 1.618 \text { for } \nu=1 .
\end{aligned}
$$

Thus, the total energy (density) is bracketed as

$$
m c^{2}+\mathcal{V} \leq \mathcal{E} \leq \beta m c^{2}+\mathcal{V} .
$$

This is the main result of the classical brane probe analysis. This extended inequality will turn out to 'screen' the singularities from the brane probe. Explicit examples will be given in section 局.

${ }^{6}$ The angular momentum is conserved, and is $\ell=\frac{m r^{2} v_{\theta}}{\sqrt{1-v^{2} / c^{2}}}=$ const. for codimension-2. 


\subsection{Semiclassical analysis}

The radial momentum may be rewritten as

$$
\wp_{r} \stackrel{\text { def }}{=} \frac{\partial \mathcal{L}}{\partial v_{r}}=\frac{m v_{r}}{\sqrt{1-v^{2} / c^{2}}}=\frac{\chi m v_{r}}{\sqrt{1-v_{r}^{2} / c^{2}}},
$$

where (in codimension-2)

$$
\chi \stackrel{\text { def }}{=}\left[1-\frac{1}{1+m^{2} c^{2} r^{2} / \ell^{2}}\right]^{-1 / 2}
$$

accounts for the (constant) angular momentum. Similarly, the total energy function (2.5) becomes

$$
\mathcal{E}=\frac{\chi m c^{2}}{\sqrt{1-v_{r}^{2} / c^{2}}}+\mathcal{V}
$$

Eliminating $v_{r}$ from Eqs. (2.15)-(2.17), we obtain

$$
-\wp_{r}^{2}+(\mathcal{E}-\mathcal{V})^{2}-\chi m c^{2}=0 .
$$

Upon substituting $\wp_{r} \rightarrow-i \hbar \partial_{r}$, this produces the Klein-Gordon equation for the brane probe's (stationary) wave-function:

$$
\partial_{r}^{2} \psi(r)+(\hbar c)^{-2}\left[(\mathcal{E}-\mathcal{V})^{2}-\chi^{2} m^{2} c^{4}\right] \psi(r)=0
$$

For future reference, the 'relativistic' WKB wave-functions for the brane probe is

$$
\psi_{ \pm}(r)=\frac{1}{\sqrt{k(r)}} e^{ \pm i \int \mathrm{d} r k(r)}, \quad k(r) \stackrel{\text { def }}{=} \frac{1}{\hbar c} \sqrt{(\mathcal{E}-\mathcal{V})^{2}-\chi^{2} m^{2} c^{4}}
$$

and for small $c$ :

$$
\begin{aligned}
c^{2} \ll \frac{(\mathcal{E}-\mathcal{V})}{\chi m} & \Rightarrow k(r) \sim \frac{(\mathcal{E}-\mathcal{V})}{\hbar c} \\
& \Rightarrow \psi_{ \pm}(r) \sim \sqrt{\frac{\hbar c}{(\mathcal{E}-\mathcal{V})}} e^{ \pm i \varphi(r)}
\end{aligned}
$$

In the case of interest, $c^{2}$ and the wave-function (2.21) both vanish at the singularity, thus preserving probability.

\section{Applications}

The above general results apply straightforwardly to several examples of recent interest, which we discuss in this section. Let us start by analyzing the BPS solution wrapped on a 


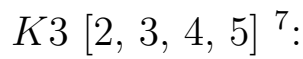

$$
\begin{aligned}
\mathrm{d} s_{s}^{2} & =Z_{p-4}^{-1 / 2} Z_{p}^{-1 / 2}\left\|\mathrm{~d} x_{\|}\right\|^{2}+Z_{p-4}^{1 / 2} Z_{p}^{-1 / 2} \mathrm{~d} s_{K 3}^{2}+Z_{p-4}^{1 / 2} Z_{p}^{1 / 2}\left\|\mathrm{~d} x_{\perp}\right\|^{2}, \\
e^{\Phi} & =g_{s} Z_{p-4}^{\frac{7-p}{4}} Z_{p}^{\frac{3-p}{4}}, \\
C_{p-3} & =\left(Z_{p-4}^{-1}-1\right) g_{s}^{-1} \mathrm{~d}^{p-3} x_{\|}, \quad C_{p+1}=\left(Z_{p}^{-1}-1\right) g_{s}^{-1} \mathrm{~d}^{p-3} x_{\|} \wedge \mathrm{d}^{4} x_{K 3} .
\end{aligned}
$$

The harmonic functions $Z_{p}$ and $Z_{p-4}$ are given as

$$
\begin{array}{rll}
Z_{p} & \stackrel{\text { def }}{=} 1+a_{p} r^{p-7}, & \text { where } \quad a_{p} \stackrel{\text { def }}{=} N g_{s}(2 \sqrt{\pi})^{1-p}\left(\sqrt{\alpha^{\prime}}\right)^{3-p} \Gamma\left(\frac{3-p}{2}\right) \\
Z_{p-4} & \stackrel{\text { def }}{=} 1-\frac{V_{*}}{V_{K 3}} a_{p} r^{p-7}, \quad \text { and } \quad V_{*} \stackrel{\text { def }}{=}\left(2 \pi \alpha^{1 / 2}\right)^{4} .
\end{array}
$$

Note that $Z_{p}\left(Z_{p-4}\right)$ is a monotonically decreasing (increasing) function: $Z_{p}(0)=+\infty$ and $Z_{p}(\infty)=1$, while $Z_{p-4}\left(r_{\mathrm{r}}\right)=0$ and $Z_{p-4}(\infty)=1$. In fact, $r_{\mathrm{r}}$ is the location of a repulsive singularity [2].

Let us now study the brane probe dynamics following section 2. From Eqs. (2.4), the warp factors $e^{2 A}, e^{2 B}$ being the metric components along $\left\|\mathrm{d} x_{\|}\right\|^{2}$ and $\left\|\mathrm{d} x_{\perp}\right\|^{2}$, and Eqs. (3.1) we have

$$
\begin{aligned}
c & =Z_{p-4}^{-1 / 2} Z_{p}^{-1 / 2} \\
m & =\tau_{p} Z_{p-4}^{+1}\left(V_{K 3}-V_{*} Z_{p} Z_{p-4}^{-1}\right) \\
\mathcal{V} & =\tau_{p}\left[Z_{p}^{-1}\left(V_{K 3}-V_{*} Z_{p} Z_{p-4}^{-1}\right)-\left(V_{K 3}-V_{*}\right)\right] .
\end{aligned}
$$

This gives us the following extended inequality for the total energy (density) of the probe

$$
0 \leq \mathcal{E}-\tau_{p}\left(V_{K 3}-V_{*}\right) \leq \tau_{p}(\beta-1) Z_{p}^{-1}\left(V_{K 3}-V_{*} Z_{p} Z_{p-4}^{-1}\right)
$$

The second inequality gives an $r$-dependent bound. As the probe approaches the singularity from afar, its total energy meets the bound at $r_{\mathrm{i}}$; see the upper left corner of Fig. 1. There, the effects omitted in the action (2.1) start taking over, rendering the analysis incomplete.

The qualitative behavior of the brane probe for codimension- 2 solutions and one class of the non-BPS solutions in codimension larger than 2 turns out to be quite similar to the case just examined. In particular, in all BPS solutions, the lower energy bound in the inequality (2.14) is constant and can be set to zero. In contrast, in non-BPS solutions, the lower energy bound depends on $r$ and there is a corresponding limit, $r_{\mathrm{o}}$, on the space in which the brane probe can move. The upper energy bound (2.14) provides a 'soft' limit, $r_{\mathrm{i}}$, where the effects omitted in the action (2.1) are no longer negligible. In all but the case we examine below, this effectively 'screens' a metric singularity.

\footnotetext{
${ }^{7}$ Our notation is as follows: $x_{\|}$shall denote coordinates parallel to the unwrapped part of the brane, and $x_{\perp}$ those that are transverse to the whole brane probe.
} 
Finally we consider a non-BPS solution along the lines of Ref. [17, 18]:

$$
\begin{aligned}
\mathrm{d} s_{E}^{2} & =D_{p}^{\frac{\delta}{\sqrt{p+1}}}\left\|\mathrm{~d} x_{\|}\right\|^{2}+D_{p}^{\frac{-\sqrt{p+1}}{7-p}} \delta\left[1-\left(\frac{r_{\mathrm{s}}}{r}\right)^{2(7-p)}\right]^{\frac{2}{7-p}}\left\|\mathrm{~d} x_{\perp}\right\|^{2}, \\
e^{\Phi}, C_{p+1} & =\text { const. }, \quad \text { where } \quad D_{p}=\left(\frac{1-\left(\frac{r_{\mathrm{s}}}{r}\right)^{7-p}}{1+\left(\frac{r_{\mathrm{s}}}{r}\right)^{7-p}}\right), \quad \text { and } \quad \delta=\sqrt{\frac{7-p}{2}} .
\end{aligned}
$$

In Fig. 1, this is labeled as the '-' solution. $D_{p}(r)$ is a monotonically increasing function from $D_{p}\left(r_{\mathrm{s}}\right)=0$ to $D_{p}(\infty)=1$. At $r=r_{\mathrm{s}}$ there is a singularity [18].

The extended inequality on the total energy (2.14) now reads:

$$
\tau_{p} D_{p}^{\frac{ \pm \delta \sqrt{p+1}}{2}} \leq \mathcal{E}-\mathcal{V} \leq \tau_{p} \beta D_{p}^{ \pm \delta \sqrt{p+1}}
$$

This limits the space available to the brane probe on both ends. The lower energy limit gives a classical turning point, $r_{\mathrm{o}}$, while the upper energy limit produces the 'soft' limit, $r_{\mathrm{i}}$, beyond which the relativistic effects omitted in the action (2.1) can no longer be neglected.

In contrast, this brane probe analysis now is reliable near $r_{\mathrm{o}}$ and $r_{\mathrm{s}}$. From Eqs. (3.7), the wave function here behaves as described in Eq. (2.21). Across $r_{\mathrm{o}}$, the standard WKB

matching conditions allow us to extend the wave function into the region $r_{\mathrm{s}} \leq r \leq r_{\mathrm{o}}$. There,

$$
\left|\psi_{ \pm}(r)\right| \sim \sqrt{c} e^{\mp \tilde{\varphi}_{0} c^{\epsilon}} \rightarrow 0, \quad \text { when } \quad r \rightarrow r_{\mathrm{s}}
$$

where $\tilde{\varphi}_{0}=$ const., and $\epsilon>0$. Since the wave function $\psi_{ \pm}$vanishes at $r_{\mathrm{s}}$, we can-and $d o$ - set it to zero for $0 \leq r \leq r_{\mathrm{s}}$. As this is where probability may leak, the vanishing of $\psi_{ \pm}(r)$ in this region ensures the conservation of probability. Our result is in agreement with the analyses in Refs. [13, 19].

\section{Conclusions}

In this note we described a relativistic probe analysis of a variety of singular spacetime geometries governed by the general structure of the Dirac-Born-Infeld action. In particular, we discussed the BPS solution wrapped on a $K 3$ manifold as well as certain non-BPS generalizations. We argued that the behavior of the semiclassical brane probe wave functions implies that unitarity boundary conditions can be imposed at the singularity. This is consistent with some existing prescriptions for the resolution of singularities [19, 20]. Although our analysis is incomplete near the singularities, we note formally that the wave functions vanish at the singularity. In addition, a more realistic description of the brane probes should take into account their energy loss to the singularity and radiation. We observe that the candidate energy function $\mathcal{E}^{\prime}=\mathcal{L}+\mathcal{V}$ has the right qualitative properties: (1) $\mathcal{E}^{\prime}$ agrees with 
$\mathcal{E}$ in the nonrelativistic regime, and $(2) \mathcal{E}^{\prime}$ vanishes at the singularity. The latter property implies that $r_{\mathrm{i}}$ becomes a hard limit, i.e., the singularity is now truly screened. Moreover, the WKB wave functions become exponentially suppressed and vanish at the singularity.

Finally, according to the generic properties of $D$-brane probes [21, 22], the resolution of the probe is $\sim \sqrt{v / c} l_{s}$. From our analysis, it then follows that $v / c \rightarrow 1$ as the brane probe approaches the singularity. Thus, the resolution of the probe at the singularity is always of the order of $l_{s}$.

Acknowledgments: We thank I. Bars, A. Brandhuber and N. Itzaki for useful discussions. The work of P. B. and D. M. was supported in part by the US Department of Energy under grant number DE-FG03-84ER40168. T. H. wishes to thank the US Department of Energy for their generous support under grant number DE-FG02-94ER-40854, and the Caltech-USC Center for Theoretical Physics where this work was completed.

\section{References}

[1] K. Behrndt: Nucl. Phys. B455(1995)188; R. Kallosh and A. Linde: Phys. Rev. D52(1995)7137; M. Cvetič and D. Youm: Phys. Lett. B359(1995)87.

[2] C.V. Johnson, A.W. Peet and J. Polchinski: Phys. Rev. D61(2000)086001, hepth/9911161.

[3] A. Buchel, A.W. Peet and J. Polchinski: hep-th/0008076.

[4] N. Evans, C.V. Johnson and M. Petrini: JHEP 0010 (2000) 022.

[5] C.V. Johnson: hep-th/0007170.

[6] C.V. Johnson: hep-th/0011008.

[7] M. Bertolini, P. Di Vecchia, M. Frau, A. Lerda, R. Marotta and I. Pesando: hepth/0011077.

[8] M. Bershadsky, C. Vafa and V. Sadov: Nucl. Phys. B463(1996)420, hep-th/9510225.

[9] P. Berglund, T. Hübsch and D. Minic: hep-th/0012042.

[10] B.R. Greene, A. Shapere, C. Vafa and S.-T. Yau: Nucl. Phys. B337(1990)1.

[11] P.S. Green and T. Hübsch: Int. J. Mod. Phys. A9(1994)3203-3227.

[12] P. Berglund, T. Hübsch and D. Minic: JHEP 0009 (2000) 015, hep-th/0005162. 
[13] A.G. Cohen and D.B. Kaplan: Phys. Lett. 470(1999)52, hep-th/9910132.

[14] E. S. Fradkin and A. Tseytlin: Phys. Lett. B163(1985)123; A. Abouelsaood, C.G. Callan, C.R. Nappi and S.A. Yost: Nucl. Phys. B280(1987)599; R. Leigh: Mod. Phys. Lett. A4(1989)2767.

[15] The general reference on $D$-branes is J. Polchinski: hep-th/9611050.

[16] E. Dudas and J. Mourad: hep-th/0004165; R. Blumenhagen and A. Font: hepth/0011269.

[17] B. Zhou and C.J. Zhu: hep-th/9903118; hep-th/9905146.

[18] M. Bertolini, P. Di Vecchia, M. Frau, A. Lerda, R. Marotta and R. Russo: hepth/0007097.

[19] R. Wald: J. Math. Phys. 21(1980)2802; G. T. Horowitz and D. Marolf: Phys. Rev. D52(1995)5670; A Ishibashi and A. Hosoya: Phys. Rev. D60(1999)104028; For an application of this criterion see A. Brandhuber and K. Sfetsos: hep-th/0004148.

[20] S. S. Gubser: hep-th/0002160; for other criteria, see J. Maldacena and C. Nunez, hep-th/0007018 and H. D. Kim, hep-th/0012091.

[21] M. R. Douglas, D. Kabat, P. Pouliot and S. H. Shenker: Nucl. Phys. B485(1997)85.

[22] T. Yoneya: Mod. Phys. Lett. A4(1989)1587. T. Yoneya: Prog. Theor. Phys. 97 (1997) 949; T. Yoneya: hep-th/0004074; M. Li and T. Yoneya: Phys. Rev. Lett. 78(1997)1219; M. Li and T. Yoneya: hep-th/9806240; D. Minic: Phys. Lett. B442(1998)102. 


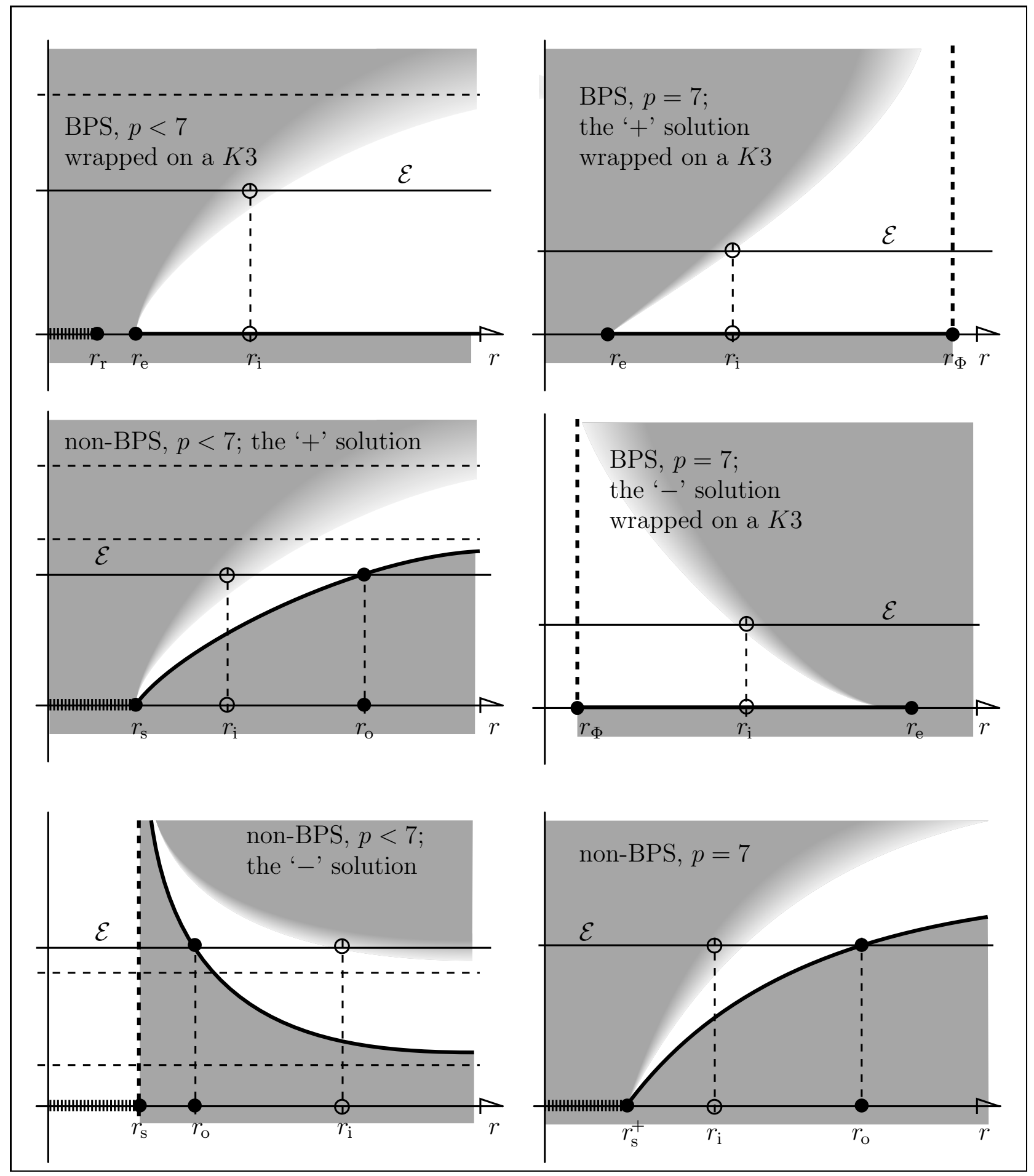

Figure 1: The energy diagram for various cases of probing spacetime singularities with branes. $m c^{2}$ becomes imaginary in the heavily dashed values of $r$. At $r_{\Phi}, e^{\Phi} \rightarrow \infty ; r_{\mathrm{s}}, r_{\mathrm{r}}$ and $r_{\mathrm{e}}$ are the naked singularity, repulson and enhançon radius, respectively. 